\title{
Cytogenetic Study in Patients with Ambiguous Genitalia
}

\section{A R T I C L E I N F O}

\section{Article Type}

Case Series

\section{Authors}

Abdi A. ${ }^{1} B S C$

Zarbati N. ${ }^{1} B S c$,

Asami M. ${ }^{1} B S C$

Bagherizadeh I. ${ }^{1} M S c$,

Hadipour $\mathrm{F}^{2} M D$,

Hadipour Z. ${ }^{2} M D$,

Shafaghati $\mathrm{Y}^{1} M D$,

Behjati F.* $P h D$

\section{How to cite this article} Abdi A, Zarbati N, Asami M, Bagherizadeh I, Hadipour F, Hadipour Z, Shafaghati Y, Behjati F. Cytogenetic Study in Patients with Ambiguous Genitalia. Sarem Journal of Reproductive Medicine. 2017;1(2):79-83.
*Sarem Fertility \& Infertility Research Center (SAFIR), Sarem Women's Hospital, Tehran, Iran ${ }^{1 " S a r e m}$ Cell Research Center (SCRC)" and "Medical Genetics Department", Sarem Women's Hospital, Tehran, Iran

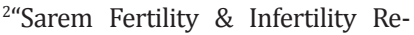
search Center (SAFIR)", "Sarem Cell Research Center (SCRC)" and "Medical Genetics Department", Sarem Women's Hospital, Tehran, Iran

\section{Correspondence}

Address: -

Phone: -

Fax: -

f_behjati@uswr.ac.ir

\section{Article History}

Received: December 22, 2015

Accepted: May 18,2016

ePublished: June 15, 2017

\section{A B S T R A C T}

Intrduction Ambiguous genitalia is a tragic event, and causes a serious emotional burden to the family. In some cases with this disorder it may cause an urgent medical problem. Precisediagnosis is mandatory for efficient intervention to help the affected patient. Chromosome analysis is the first step in evaluation of the patients with ambiguous genitalia. A chromosomal study is conducted to determine the sex of patients with sexual ambiguity using standard methods. In this method, peripheral blood lymphocyte cells were cultured in a complete RPMI medium, and a high-resolution GTG banding technique was used for studying, and at least 20 chromosomal amplitudes were studied.

Patients Information Two patients were 46XY and two others had 46XX karyotype. Patients with 46XX karyotype had Congenital Adrenal Hyperplasia (CAH). The ovarian and uterine tissues were normal in them, but they had a ambiguous external male genitalia. In the study of hormones of one of these patients, the amount of 17-hydroxyprogesterone and cortisol was normal, and high levels of aldosterone and potassium were reported. Patients with 46xy karyotype have Complete Androgen Insensitivity Syndrome (CAIS) and have a female female reproductive system. There are no uterine tubes in this group of patients and the testicles are in the abdomen.

Conclusion Karyotype performance is essential for patients with ambiguous genitalia, in order to investigate the relationship between phenotype and genotype and thus the correct management of patients.

Keywords Ambiguous Genitalia; Chromosome Abnormality; Congenital Adrenal Hyperplasia; Complete Androgen Insensitivity Syndrome 


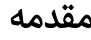

تعيين اوليه جنسيت هنگام لقاح صورت مىگيرد و يروسه فنوتييى

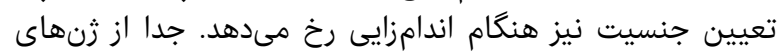
خاص جنسى روى كروموزوم X

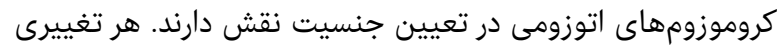

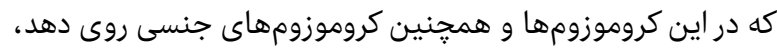

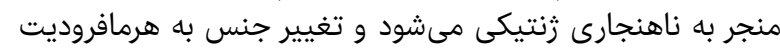

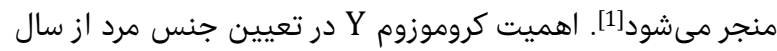

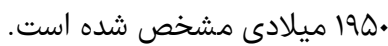

فاكتور اصلى تعيين جنسيت زنتيكى يك جنى جنين، با حضور يا عدم

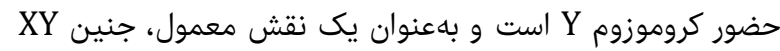
مرد و جنين XX زن ميى شود. اطلاعات مبنى بر شيوع و نفوذ دلايل

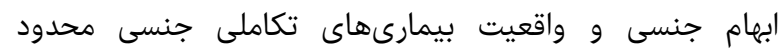

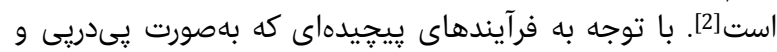

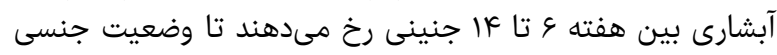

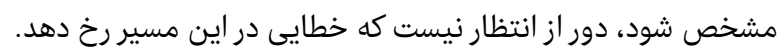

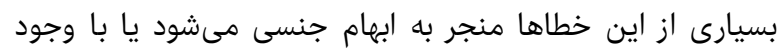

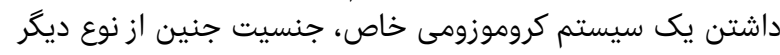

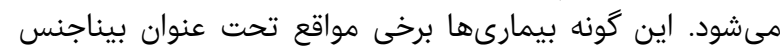

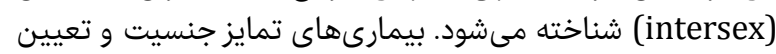

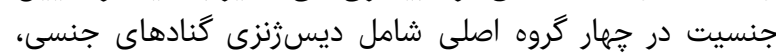

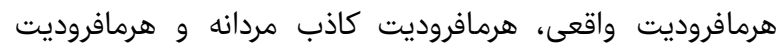

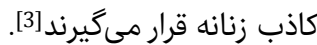

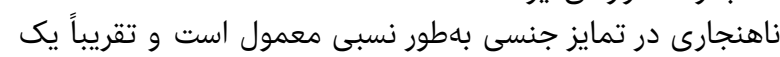

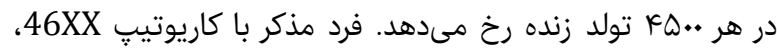

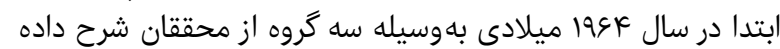

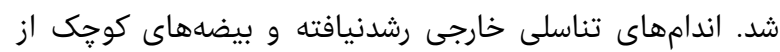

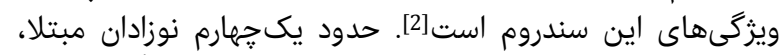

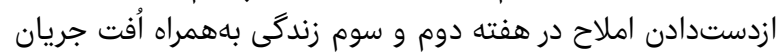

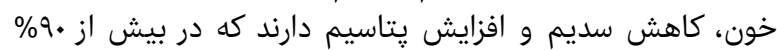

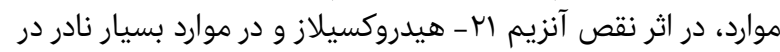

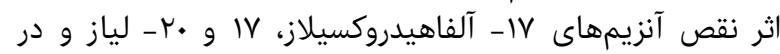

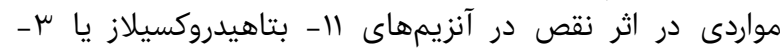

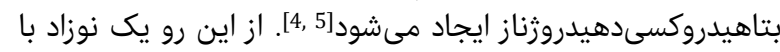

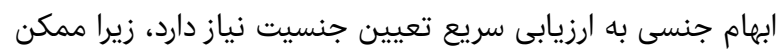

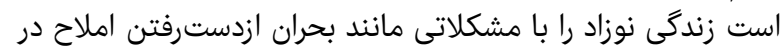

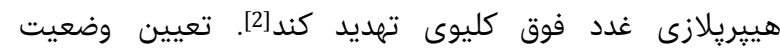

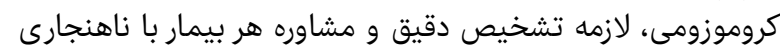

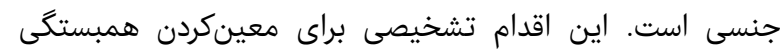

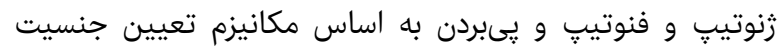
ضرورى است.

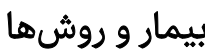

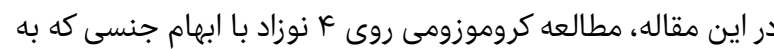

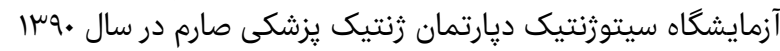

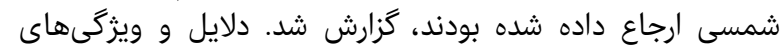

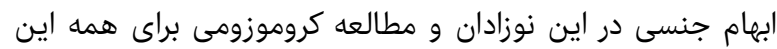
بيماران صورت كرفت. انجام كارئ نوزان ناهنجارى هاى كروموزومى كمك كند.

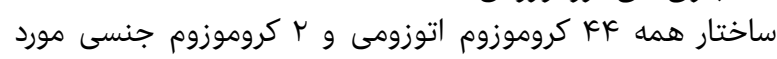

مطالعه سيتوزنتيك در جهار نوزار ارئاد ارجاعشده با

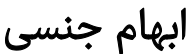

اكرم عبدى BSc

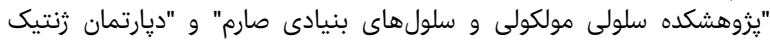
يزشكى صارم"، بيمارستان فوق تخصصى فول صارم، تهران، ايران مان

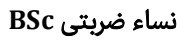

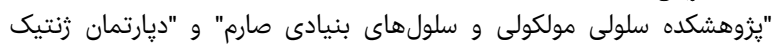

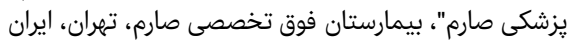

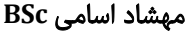

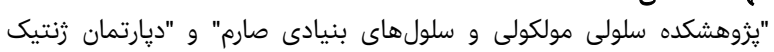

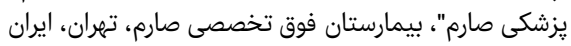

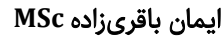

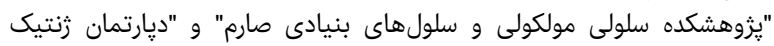

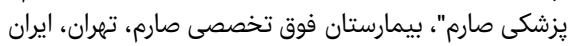

فاطمه هادى يور

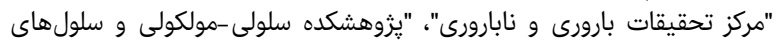

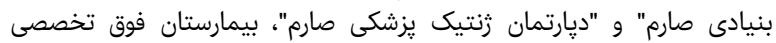

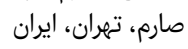

MD زهرا هادى نهران، ايران

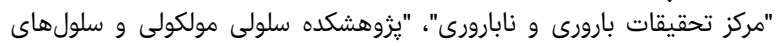

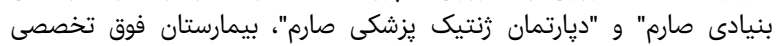

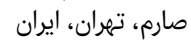

يوسف شفقتى تصني ايران

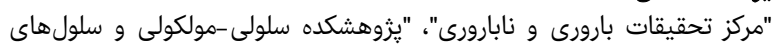

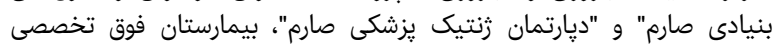

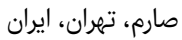

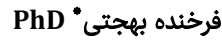

"مركز تحقيقات بارورى و نابارورى"، بيمارستان فوق تخصصى صارم، تهران، ايران

جكيده

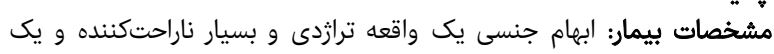

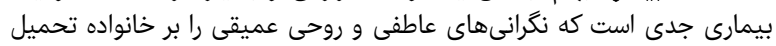

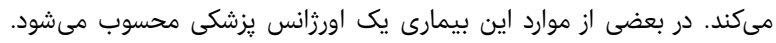

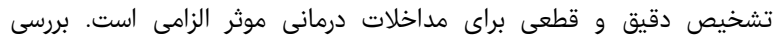

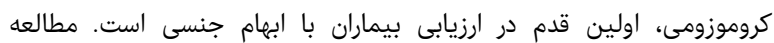

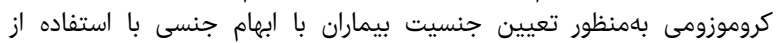

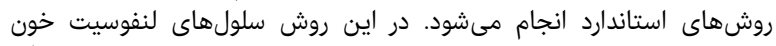

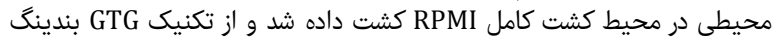

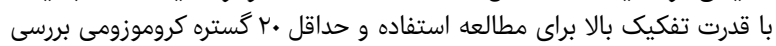

دو بيمار، كاريوتيب 46XX و دو مورد 46XY را داشتند. بيمارانى كه كاريوتيڤ

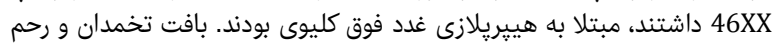

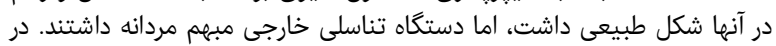

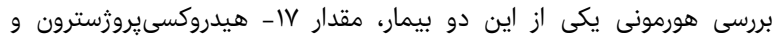

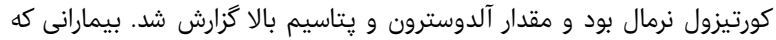
كاريوتيڤ

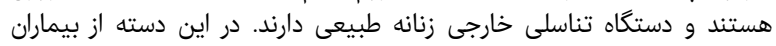

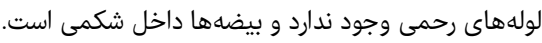

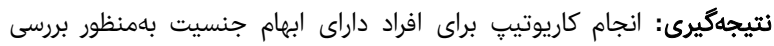

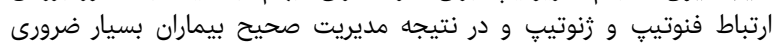

كليدوازهها: ابهام جنسى، ناهنجارى كروموزومى، هيبيريلازى غدد فوق كليوى، سندروم

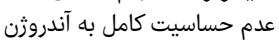

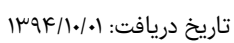

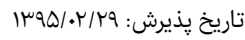

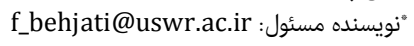


جنسى، تستيكولار فمينيزيشن نسبت داده شده است كه دليل داديل

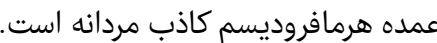

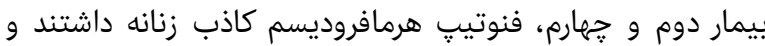

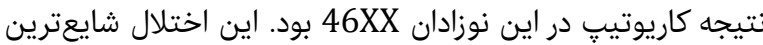

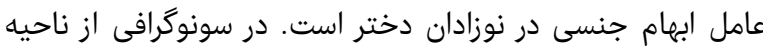

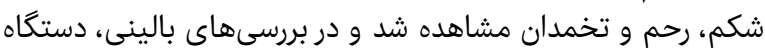

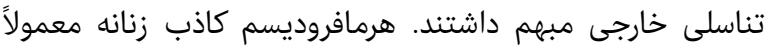

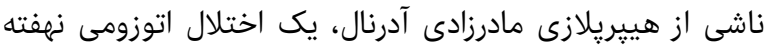

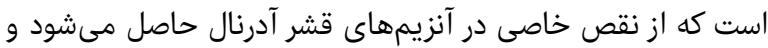

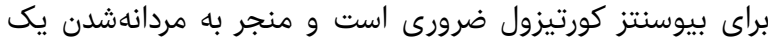

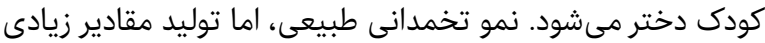

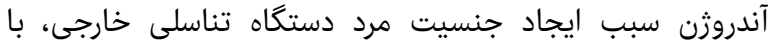

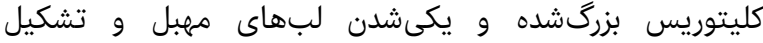

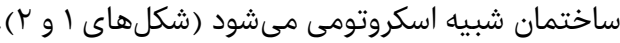

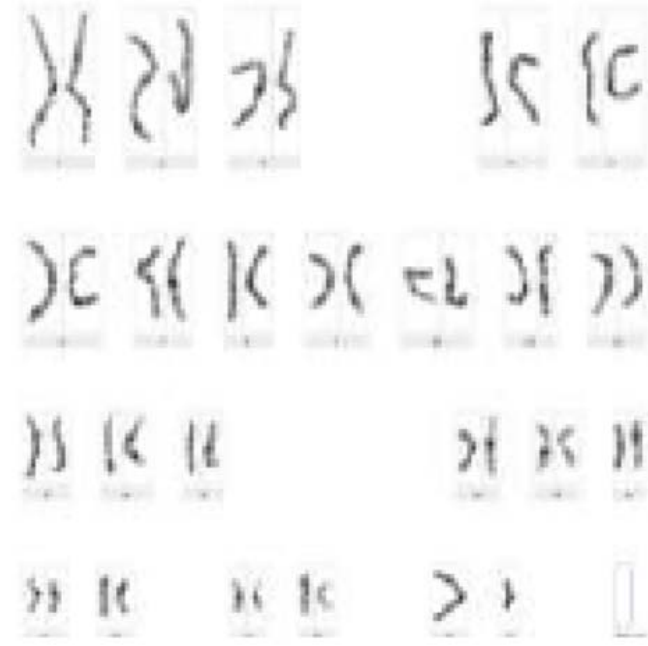

شكل () كاريوتايي بيمار ا

\section{$k \partial 2<{ }^{8}<<$ $\rightarrow 3 \|) \|>13 x$}

Si $\{6>$ ?

74 it $\rightarrow 1$

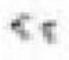

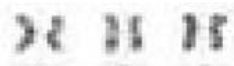

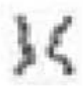

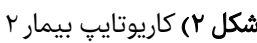

مورد سوم ابهام جنسى با بيضه دوطرفه بود و نتيجه كاريوتيڤ

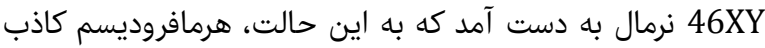

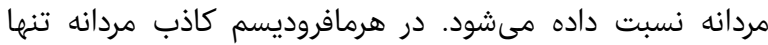

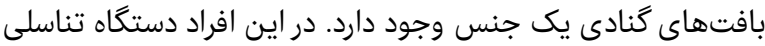

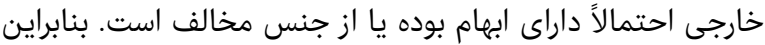

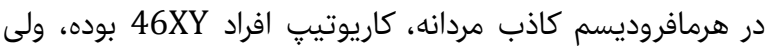

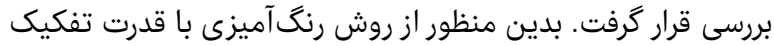

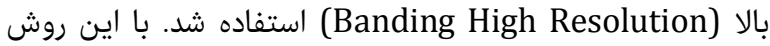

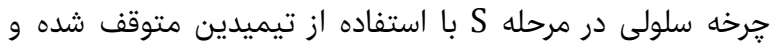

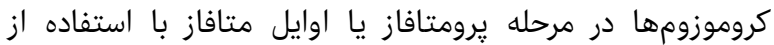

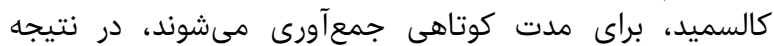

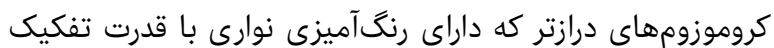

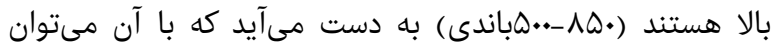

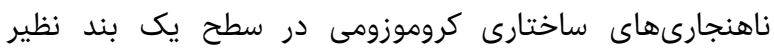

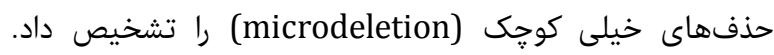

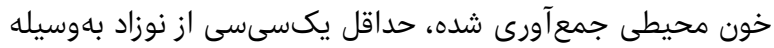

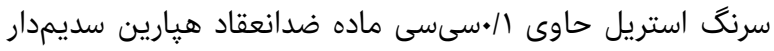

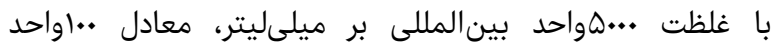

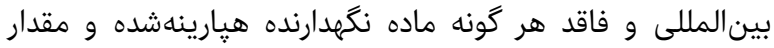

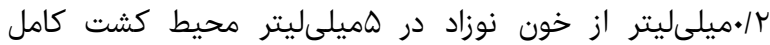

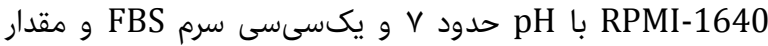

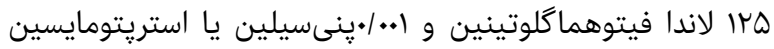

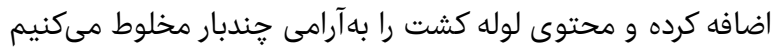

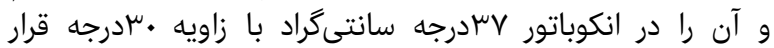

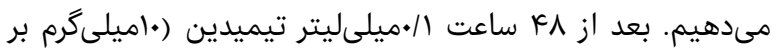

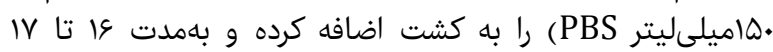

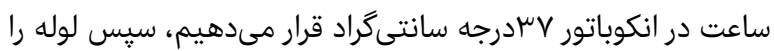

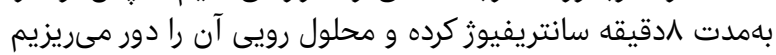

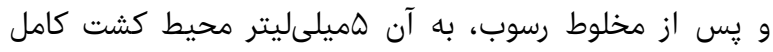

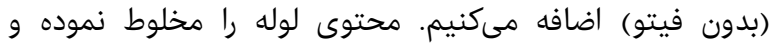

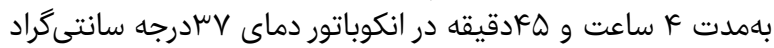

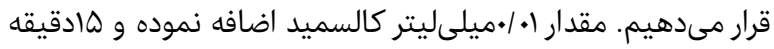

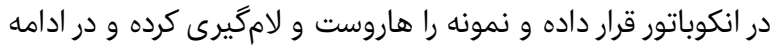

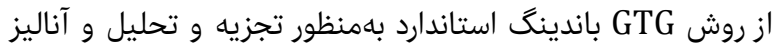

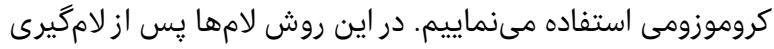

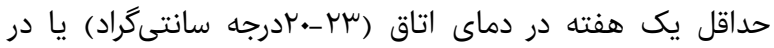

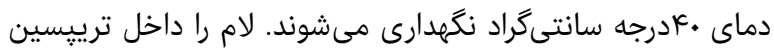

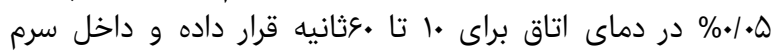

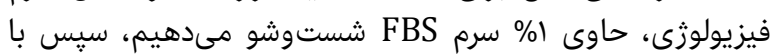

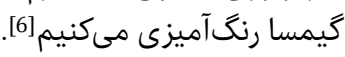

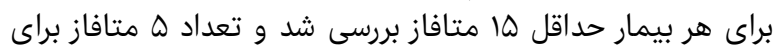

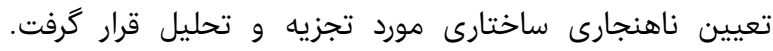

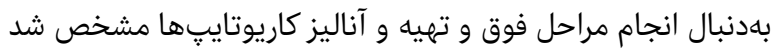

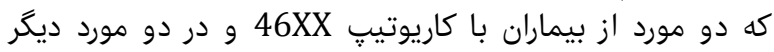
كاريوتيڤ 46XY نرمال بود (جدول ().

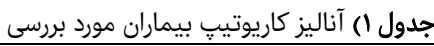

\begin{tabular}{|c|c|c|c|c|}
\hline كاريوتيب & فنوتيب & اندام تناسلى خارجى & سن (روز) & بيمار \\
\hline $46 X Y$ & مردانه & ابهام تناسلى & 1. & \\
\hline $46 X X$ & مردانه & ابهام تناسلى & $\mu$ & r \\
\hline $46 X Y$ & زنانه & ابهام تناسلى & $\wedge$ & \\
\hline $46 X X$ & مردانه & ابهام تناسلى & 1 & f \\
\hline
\end{tabular}

مورد اول نتيجه ازدواج فاميلى درجه سوم دخترعمه و يسردايى بود.

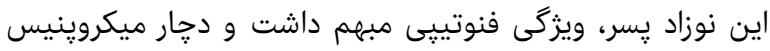

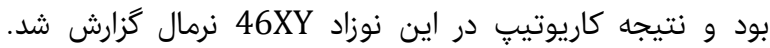

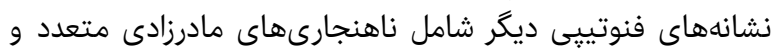

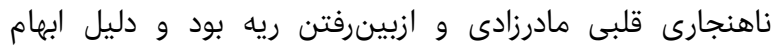


دي اكرم عبدى و همكاران

$|(\partial)| x()$

n) \|\| is $\| \geqslant$

113 in

382116

if It

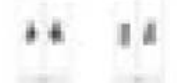

>)

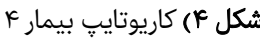

نتيجه يرى

براساس اين مطالعه، در همه اين نوزادان با ابهام جنسى و وتئ

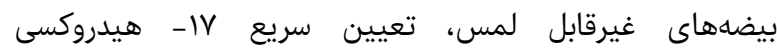

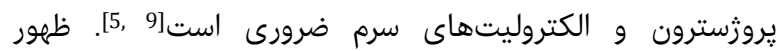

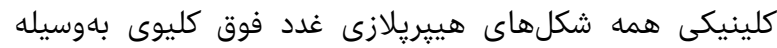

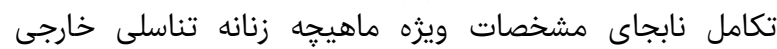

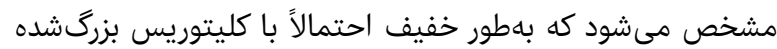

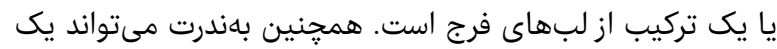

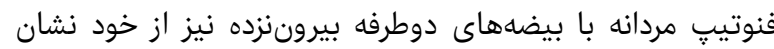

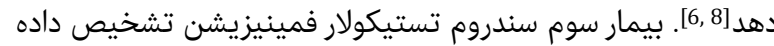

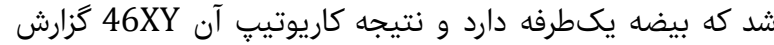

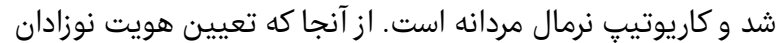

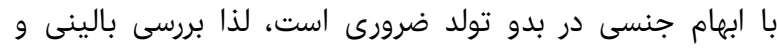

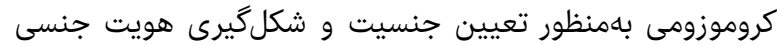

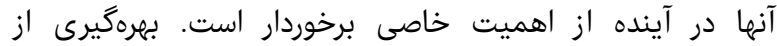

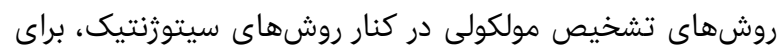

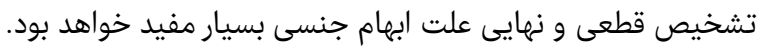

تشكر و قدردانى: از كليه همكاران ديارتمان زنتيك بيمارستان فوق تخصصى صارم تشكر مى شود.

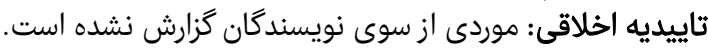

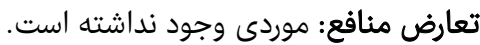

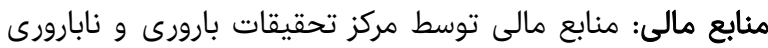

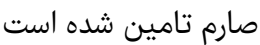

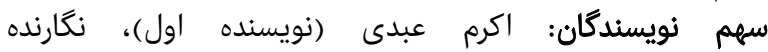

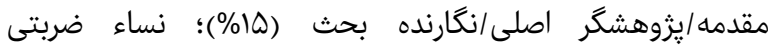

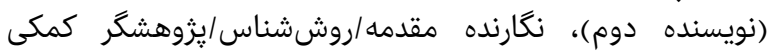

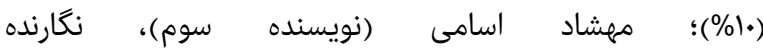

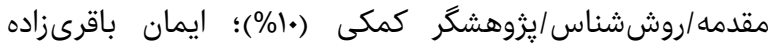

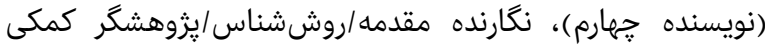

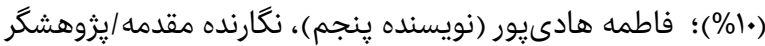

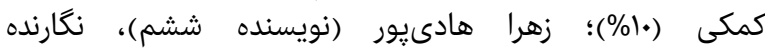

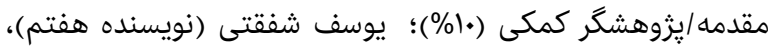

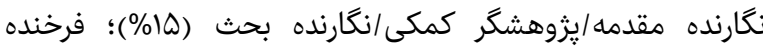

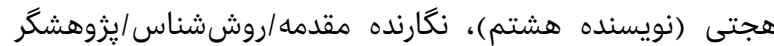

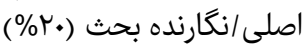

دستخاه تناسلى آنها زنانه است. عمدهان عاذرين دليل بروز هرمافروديسم

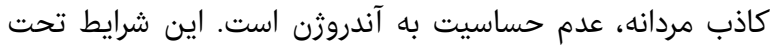

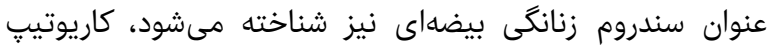

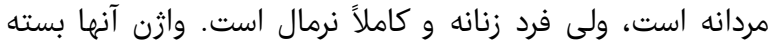

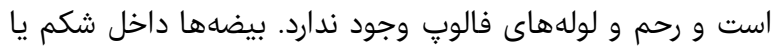

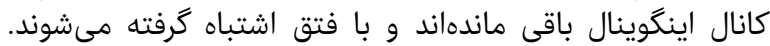

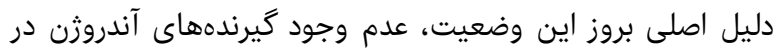

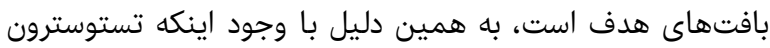

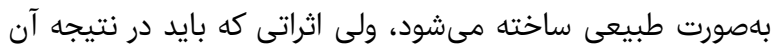

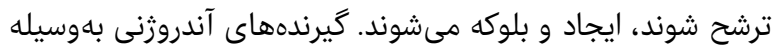

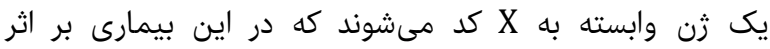

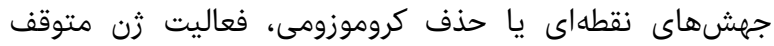

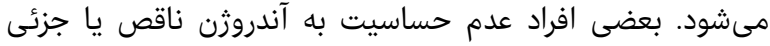

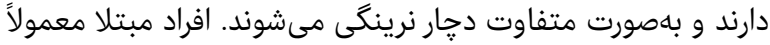

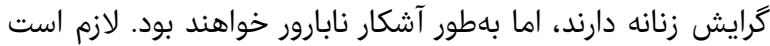

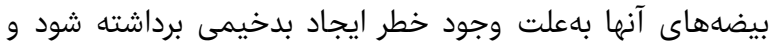

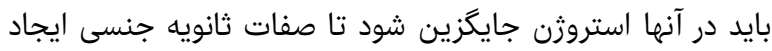

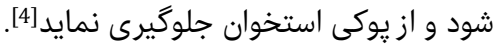

بحث

دلايل ابهام جنسى، متغير و تعيين جنسيت در بعضى از موارد

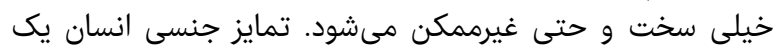

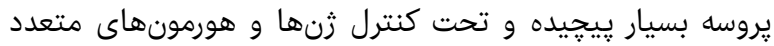

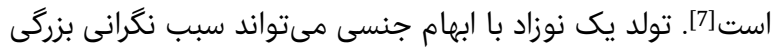

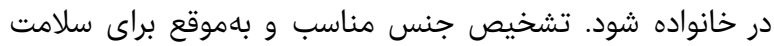

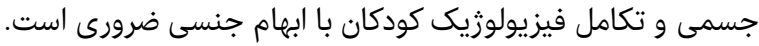

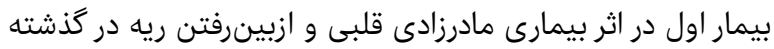

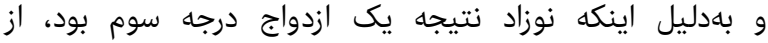

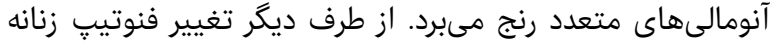

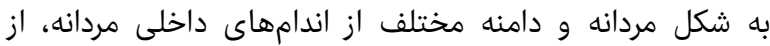

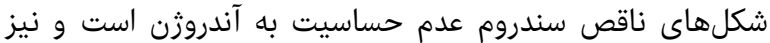

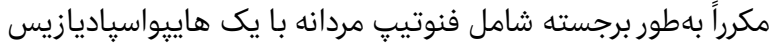

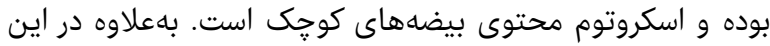

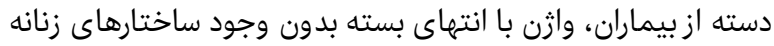

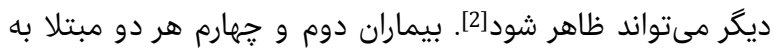

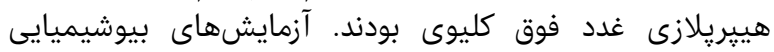

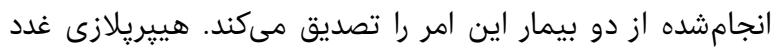

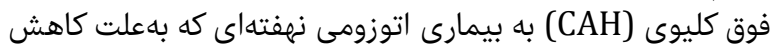

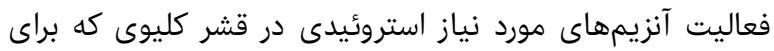

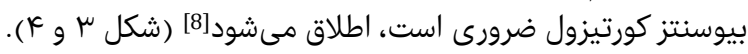

\section{$><x$ iा 11 iा}

\section{If if 11 औो}

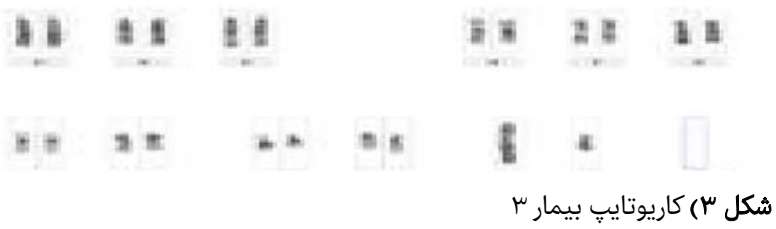


مطالعه سيتورنتيك در جهار نوزاد ارجاعشده با ابهام جنسى

6- Behjati F. Laboratory manuals in human cytogenetics. Tehran: University of Social Welfare And Rehabilitation Sciences Press; 2007. p.166. [Persian]

7- Iqbal MA. Cytogenetics and etiology of ambiguous genitalia in 120 pediatric patients. Ann Saudi Med. 2004;24(5):368-72.

8- Reynoso De Mendoza S, Vargas-González R, CamachoGutiérrez S, Marroquin-Garcia I, Chatelain-Mercado S, Sierra-Pineda F. Disorders of sexual development in genetic pediatrics: Three different ambiguous genitalia cases report from hospital Para el Nino Poblano, Mexico. Int J Gen Mol Biol. 2010;2(10):207-16.

9- Güney Y, Andrieu MN, Hiçsözmez A, Aktaş C, Kurtman

C, Gökçe ȘÇ. Seminoma in a case of testicular feminization syndrome: Patient outcome after 7 years of follow-up. Acta Oncol Tur. 2008;41(1):57-8.

1- Kaur A, Mahajan S, Singh JR. Cytogenetic analysis in cases with sex anomalies. Int J Hum Genet. 2004;4(3):167-71.

2- Yüce H, Etem E, Özbey Ü. Cytogenetic and molecular evaluation of ambiguous genitalia in pediatric patients. Firat Tıp Dergisi. 2008;13(1):28-31.

3- Chavhan GB, Parra DA, Oudjhane K, Miller SF, Babyn PS, Salle FLP. Imaging of ambiguous genitalia: Classification and diagnostic approach1. Radiographics. 2008;28(7):1891-904.

4- Turnpenny PD, Ellard S. Emery's Elements of Medical Genetics. Edinburgh: Elsvier Churchill Livingstone; 2011. 5- Nussbaum RL, McInnes RR, Willard HF. Thompson \& Thompson Genetics in Medicine. 7th Edition. Amsterdam: Elsvier. 2007. 